\title{
OCliniral hamarks
}

ON

\section{THE TREATMENT OF INVETERATE PRURITUS ANI.}

By Sir CHARLES BALL, M.CH., F.R.C.S.I., HoN. F.R.C.S.EN(i.,

Surgeon, Sir Patrick Dun's Hospital ; Regius Professor of Surgery, University of Dublin; Honorary Surgeon to the King iu Ireland.

Ar the recent meeting of the British Medical Association at Oxford an interesting discussion took place in the section of Dermatology on the causation and treatment of pruritus ani ; any one reading that debate cannot fail to be struck with the enormous number of distinct diseased. conditions to which this annoying symptom was attributed by the various speakers, and large as the number of supposed causes assigned were, the various plans of treatment advocated were still greater. All the resources, not only of the Pharmacopoeia, Sut of the Extra Pharmacopoeia, being in turn favoured.

The conclusion which naturally presents itself from reading this discussion is that the etiology of pruritus ani is in many cases indefinite, of the many causes assigned few are obviously operative, while on the other hand, the majority are purely speculative; also that the treatment is frequently eminently unsatisfactory.

Cases in which itching of the anus is the prominent symptom may be conveniently classed in three groups :

1. Those due to parasites either animal or mycotic.

2. Those resulting from dermatitis of the cutaneous portion of the anal canal and surrounding skin.

3. Those in which the disease is essentially in the nerves supplying the affected area with sensation.

\section{Parasites.}

The treatment of cases included in the first group is simple and effective once the nature of the parasitic cause has been determined.

In the second group are included cases classed under that protean generic term eczema. As in other parts of the body, great variety is to be observed in the intensity of the inflammation, the character of the local appearances, and the efficacy of treatment adopted; some cases yield to the simplest remedies, while others prove intractable to all usual therapentic methods. It will generally be found that the most suitable applications to eczema of the anal region when acute and of the moist variety are those which are emollient and unirritating, while in the more chronic and in the dry variety highly stimulating applications, such as nitrate of silver, etc., are indicated. I have frequently found the best application to be an ammonia soap, which may easily be prepared by diluting linimentum ammoniae B.P. with olive oil as much as may be necessary, but no general treatment is invariably suitable: what cures one will make another, which as far as appearances go is identical, much worse; so that it is sometimes necessary to change the treatment very frequently until the necessities of the individual case are at last met. In the treatment of these cases it must always be remembered that the local trouble may be due to some more general disease, notably gout or diabetes.

Although the majority of cases of pruritus ani due to chronic dermatitis are curable, if sufficient care and time is devoted to their treatment, a few appear to defy all the usual forms of therapeutics, and may justly be termed inveterate.

\section{Nerve Lesions.}

The third group consists of those cases in which chronic intolerable itching is present, without obvious evidence of dermatitis, although abrasions of the skin may be present, the result of scratching; there are, however, often present very definite trophic changes in the skin, notably white patches, due to loss of normal pigment, while sometimes the skin surrounding the anus is of a dull bluish-white colour with a smooth surface like parchment. That their cause is due to some change in the sensory nerves of the affected area there cannot be any doubt, but by calling them neuroses we do not advance the etiology any further than when we similarly describe certain forms of trigeminal neuralgia, the intimate pathology of which is unknown, as neuroses. In some of these cases a condition of dermatitis is present at the commencement, which, however, may quite disappear, with no improvement, but rather an aggravation of the itching; in others no inflammatory state of the skin other than that produced by scratching is ever present. These cases are quite incurable by any form of external application or internal treatment, and their lot is a very miserable one, the intolerable or, as it has been aptly termed, painful itching keeping the patient awake at night and rendering life almost unbearable.

The surgical measures which have hitherto been adopted in these cases, whether resulting from inveterate dermatitis or nerve lesion, appear to be two:(1) The cauterization of the skin affected down to the papillary layer with the benzolin cautery strongly advocated by the late sir W. Mitchell Banks, and (2) the complete removal by excision of the implicated skin. I have no personal experience of either method, as they appeared to me both crude and unscientific.

Reasoning from the analogy of the satisfactory results obtained by the removal of the Gasserian ganglion in cases of extreme trigeminal neuralgia. it occurred to me that inveterate pruritus ani might be similarly treated by the excision of the pnsterior roots and ganglia of the third and fourth sacral nerves; as, however, I feared that possibly this might interfere with sphincteric control of the rectum, I determined to adopt in the first instance the more simple procedure of division of the terminations of the sensory nerves to the affected skin. This appears such an obvious method that I feel sure it must have occurred to other surgeons, but I have not seen any cases published in which it has been carried out.

In the erect or recumbent position the anus is a canal about I in. in length, the lower two-thirds of which is covered by thin skin arranged in vertical folds, while the upper third is covered by mucous membrane of the rectum; these two are separated by a somewhat irregular line covered by transitional epithelium, where the anal valves are situated. When the nates are forcibly separated, or when the patient makes an expulgive effort, the vertical folds of skin become apparent as radiating folds, with which we are familiar when examining theanus, and the muco-cutaneous junction can be brought into view by gently separating the parts still further. The cutaneous portion of the anal canal is abundantly supplied with sensory nerves, in the terminations of which end-bulbs have been demonstrated by several observers, but that part of the anal canal which is covered by mucous membrane is, on the contrary, almost devoid of ordinary nerves of sensation; pruritus ani, therefore, never passes to the mucous membrane above the muco-cutaneous junction, but is confined to that portion of the anal canal with cutaneous covering, and the skin in the immediate neighbourhood of the anus, especially those parts which are in contact in a position of rest. If, therefore, a typical case of pruritus ani is examined, it will be found that the affected area implicates the lower portion of the anal canal and the surrounding skin, extending further in the middle line towards the coccyx behind, and along the perineum in front, even not infrequently involving the fourchette in the female, than it does upon the sides. The implicated region is elliptical in shape, quite twice as long in the antero-posterior direction as it is broad in the lateral, with the anal canal in the centre; it receives its sensory nerves from branches of the third and fourth sacral nerves, which come down on the levator ani muscle and reach the skin by perforating the external sphincter muscle. The procedure about to be described is devised for the purpose of dividing all these nerve twigs before they reach the skin of the affected area.

\section{Opration.}

The skin having been cleansed as completely as possible and shaved, a curved incision is made on each side of the affected area (Fig. I) enclosing the entire ellipse with the exception of a narrow neck in front and behind; these incisions are carried down to the sphincter muscle, and the flaps raised by careful dissection with scissors from the surface of the muscle, round its anal margin, and up the anal canal to above the muco-cutaneous junction, the dissection extending round the entire circumference, all connexions with the subjacent tissues being divided (Fig. 2). The pedicles in front and behind are now undercut to a point well beyond the area of irritation, and the outer concave edges of the incision also undercut to a distance of at least a quarter of an inch free of the involved skin all round. Care must next be taken to stop all bleeding, and the flaps should not be replaced until it is completely arrested, as the formation of a haematoma in the wound might compromise the vitality of the flaps. The flaps are finally replaced and retained by sutures, a few intervals being left between them for drainage. 
The immediate result of this operation is to render the entire ellipse included between the incision, the pedicles, and outer edges as far as the $y$ have been undercut, superficially anaesthetic (Fig. 3) and th itching is at once relieved. :

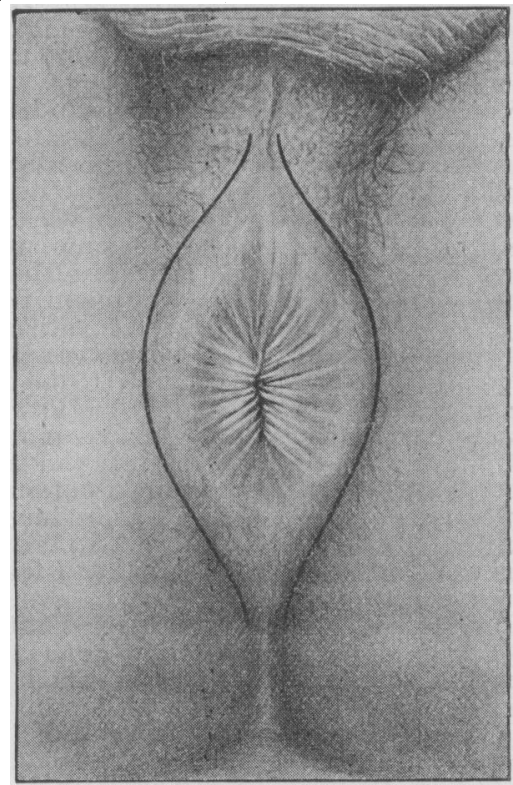

Fig. 1. - The area involved in inveterate pruritus ani, and the lines of incision in the operation described in the text.

The first case operated on in this manner occurred nearly two years ago; except for slight superficial vesication upon one side of the flap, healing was rapid; cutaneous sensation returned some months after the operation, but by this time the skin had become so altered that the pruritus did not return, and he has not had a moment's trouble with itching since. His case had been an extremely severe one, of sixteen years' duration, and his life so miserable that he threatened suicide if he could not be relieved.

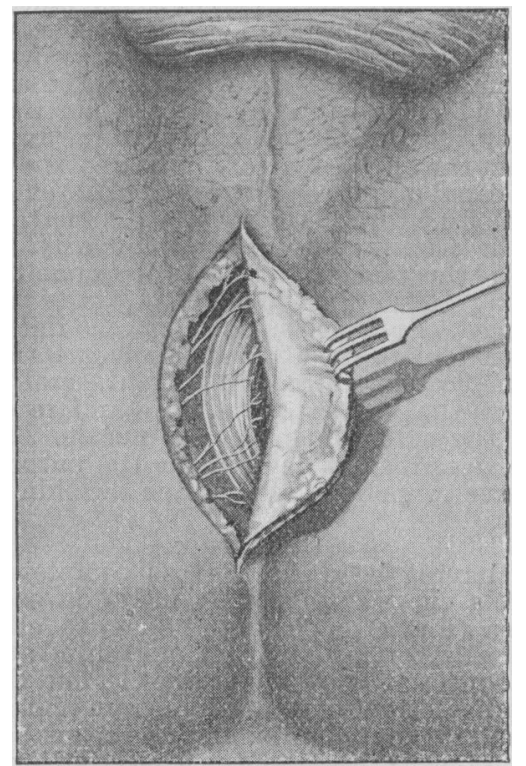

Fig. 2.- Operation for iuveterate pruritus ani. method of dissecting wh the flap and of dividing the terminal cutaneous nerve twig:, exaggerated.

In a second case, operated on thirteen months ago, the flaps united at once without trouble, and complete relief followed with the exception of one point about the size of the tip of the little finger, near the coccyx, which had not been sufficiently undercut. Five months afterwards he returned, asking that this part might be operated on as well. The raising of a flap containing the irritable area completely cured him and he l as remained well since.
In a third case, a woman, the area of irritation, besides implicating the anal region, extended forwards to the posterior fourchette, the undercutting of the anterior pedicle and skin of the perineum was therefore somewhat extensive, and was carried round the posterior margin of the vulva. Recovery in this case was rapid and the relief of the pruritus complete.
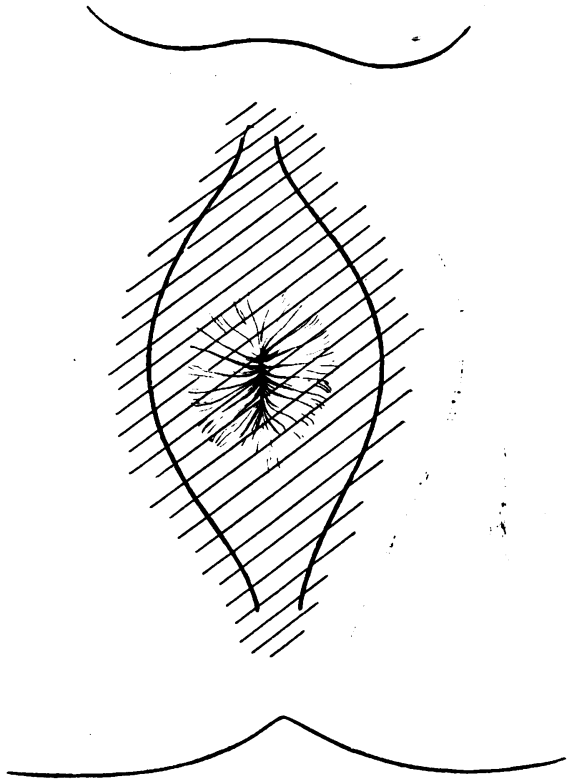

Fig. 3. The oblique Jines indicate the area which is rendered anaesthelic by the operation for inveterate pruritus ani.

So far the cases are too few to generalize from ; they, however, prove (I) that the operation gives immediate relief, and (2) that superficial sensation may be restored without recur rence of the pruritus, and we may hope that in some of these distressing cases a permanent cure will be established; we know, however, that in cases of neurectomy for trigeminal neuralgia, recurrence sometimes takes place after long intervals, and that the removal of the Gasserian ganglion alone can be considered a radical operation. Should recurrence of pruritus supervene after the operation I have described the removal of the posterior roots of the third and fourth sacra nerves with their ganglia should, I believe, be seriously considered.

\section{Remarks}

ox

\section{THE TREATMENT OF APPENDICITIS,} WITH AND WITHOUT OPERATION.

Read before the Rhyl and Denbigh Division, North Wales and Shropshire Branch.

By RUSHTON PARKER, M.B., B.S., F.R.C.S., Professor of Surgery, University of Liverpool ; Surgeon, Liverpool Royal Infirmary.

ThE occurrence of sudden and disabling pain in the right iliac fossa, with tenderness in the immediate region of the appendix, and vomiting, is ordinarily a malady of striking distinctness, not more conspicuous than its tendency to subside, and even to pass away altogether, sometimes without leaving any trace. The occurrence of relapses, however, and other circumstances have led to the practice of removing the appendix, and to the performance of operations which are among the most important, not to say interesting, in surgers. Not only has surgical operation, more readily and promptly performed, got rid of a great deal of misery in relapsing cases, and in addition saved the lives of persons whose severer cases would have been fatal without it, but it has also thrown light on the pathological anatomy of this disease much more abundantly and satisfactorily than post-mortem examinations ever can, by resson of revealing the changes in numerous cases that recover. It has thus made clinical judgement more easy, and has added enormously to our knowledge. 